July 1998

DFUB-98-16

\title{
Scaling Functions in the Odd Charge Sector of Sine-Gordon/Massive Thirring Theory
}

\author{
G. Feverati円, F. Ravaninif and G. Takács [ \\ INFN Sezione di Bologna - Dipartimento di Fisica \\ Via Irnerio 46 \\ 40126 Bologna, Italy
}

\begin{abstract}
A non-linear integral equation (NLIE) governing the finite size effects of excited states of even topological charge in the sine-Gordon (sG) / massive Thirring (mTh) field theory, deducible from a light-cone lattice formulation of the model, has been known for some time. In this letter we conjecture an extension of this NLIE to states with odd topological charge, thus completing the spectrum of the theory. The scaling functions obtained as solutions to our conjectured NLIE are compared successfully with Truncated Conformal Space data and the construction is shown to be compatible with all other facts known about the local Hilbert spaces of $\mathrm{sG}$ and $\mathrm{mTh}$ models. With the present results we have achieved a full control over the finite size behaviour of energy levels of $\mathrm{sG} / \mathrm{mTh}$ theory.
\end{abstract}

\section{Introduction}

Finite size effects are widely recognized to play a major role in modern QFT (Quantum Field Theory) [1]. The concept of scaling functions, encoding the dependence of energy levels on the finite volume (Casimir effect), has shown its practical convenience for a control of renormalization flows under change of scale of many QFTs. Most of the progress made so far in computing the scaling functions including non-perturbative effects have dealt with twodimensional QFT, in particular with integrable QFTs, where exact methods à la Bethe Ansatz can be used to diagonalize Hamiltonians. A control of the behaviour of energy levels of a two-dimensional integrable QFT under change of the finite volume of a cylinder space-time geometry can lead not only to useful renormalization flow information, but also serves as a basis for future developments in the investigation of correlation functions [2].

A method of investigation that has been very fruitful, even for theories which are not integrable, is the Truncated Conformal Space (TCS) approach [3], which is an intrinsically non-perturbative method, but has problems of principal nature, coming from the fact that one does not have an analytic control of the spectrum, and of practical nature, because to reach a certain precision in the resulting energy levels one has sometimes to resort to very high truncation levels and introduce enormous matrices to diagonalize.

\footnotetext{
${ }^{1}$ E-mail: feverati@bo.infn.it

${ }^{2}$ E-mail: ravanini@bo.infn.it

3 E-mail: takacs@bo.infn.it
} 
For integrable QFT, there also exist exact analytic methods to compute the finite size effects, like e.g. the Thermodynamic Bethe Ansatz (TBA) 《4. Another approach is the nonlinear integral equation (NLIE) introduced as a continuum limit of Bethe equations emerging from a light-cone lattice regularisation, by Destri and De Vega [5, 6]. (Similar equations had already appeared in a different condensed matter context in [0]). These methods provide an impressive numerical precision of the scaling functions, compared to the TCS one, and also give an analytic control. The principal limitation of these exact methods was basically that only the vacuum (and a few excited states degenerating with it at $L \rightarrow \infty$ ) could be obtained. However, progress of the last few years have opened the investigation to excited states (see e.g. for the TBA approach refs. [8, 9]). In the Destri-De Vega approach, the excited state equation was introduced in [10] and later generalized to include generic configurations of Bethe roots in [11]. The correct quantisation rule was established in [12, 13], where it was shown that the resulting scaling functions agree with numerical TCS data.

The power of the NLIE method lies in the fact that it allows to describe all excited states in a single framework for all values of the sG coupling constant, and it can be used even in cases when the vacuum TBA is not known (in contrast with the TBA approach of [8, 9]). It was argued in [10] that a so-called $\alpha$-twist following the ideas of Zamolodchikov [14] gives an access to scaling functions in $\Phi_{(1,3)}$ perturbations of minimal models, the simplest examples of which include the scaling Yang-Lee and the Ising model in zero magnetic field.

The NLIE still has a serious limitation, namely that only states lying in sectors with even topological charge can be accessed. This restriction is an apparently unavoidable feature of the light-cone lattice construction from which the NLIE is deduced. However, it is of primary importance to master also the scaling functions of the states with odd topological charge, as for example the single soliton state in $\mathrm{sG}$, or the single fermion state in $\mathrm{mTh}$. In this letter we give a conjecture of how the NLIE looks for such odd topological charge states, starting from the results for the UV conformal dimensions computed in 13. The solutions of the NLIE proposed for the odd sector are then compared to the TCS approach for $\mathrm{sG} / \mathrm{mTh}$, i.e. perturbed $c=1 \mathrm{CFT}$ that we have developed in [12, 13]. Finally, the possibility to twist the NLIE itself leads us to a full control of all periodic/antiperiodic boundary conditions. This allows us to describe the differences between mTh and sG that should be expected in the odd charge sectors, in full accordance with the ideas of [15].

Throughout the paper we adhere to the same notations as in [12, 13]. The reader is invited to consult those papers (and references therein) as an introduction to the concepts and general setup.

\section{UV analysis in the NLIE framework}

The NLIE describing the excited state spectrum of $\mathrm{sG} / \mathrm{mTh}$ theory for even value of the topological charge can be deduced from an inhomogeneous six-vertex model put on a lightcone lattice and has the following form in the continuum limit

$$
Z(\vartheta)=\mathcal{M} L \sinh \vartheta+g\left(\vartheta \mid \vartheta_{j}\right)+2 \Im m \int_{-\infty}^{\infty} d x G(\vartheta-x-i \eta) \log \left(1+(-1)^{\delta} e^{i Z(x+i \eta)}\right),
$$

where $L$ is the spatial volume and $\mathcal{M}$ is the soliton mass. We also introduce the dimensionless volume parameter $l=\mathcal{M L}$. The function $g\left(\vartheta \mid \vartheta_{j}\right)$ is the so-called source term, composed of the contributions from the holes, special objects and complex roots (close and wide) which we 
call sources and denote their positions by the general symbol $\left\{\vartheta_{j}\right\}=\left\{h_{k}, y_{k}, c_{k}, w_{k}\right\}$ and is

$$
g\left(\vartheta \mid \vartheta_{j}\right)=\sum_{k=1}^{N_{H}} \chi\left(\vartheta-h_{k}\right)-2 \sum_{k=1}^{N_{S}} \chi\left(\vartheta-y_{k}\right)-\sum_{k=1}^{M_{C}} \chi\left(\vartheta-c_{k}\right)-\sum_{k=1}^{M_{W}} \chi\left(\vartheta-w_{k}\right)_{I I},
$$

where the function $\chi$ is the soliton-soliton phaseshift in sG theory, $G$ is its derivative up to a factor of $2 \pi$, and the index $I I$ denotes the second determination necessary for wide roots (for details see [13]). The positions of the sources are related to the counting function $Z(\vartheta)$ by the Bethe Ansatz quantisation conditions

$$
Z\left(\vartheta_{j}\right)=2 \pi I_{j}, I_{j} \in \mathbb{Z}+\frac{1-\delta}{2},
$$

where $I_{j}$ are the Bethe quantum numbers. There is a so-called counting equation

$$
N_{H}-2 N_{S}=2 S+M_{C}+2 \theta(\pi-2 \gamma) M_{W},
$$

relating the number of the different roots/holes to $S$, which is the spin of the XXZ chain appearing in the lattice regularisation and takes integer values for the Bethe states. The sG topological charge $Q$ turns out to be $Q=2 S$.

Once the solution for the counting function $Z(\vartheta)$ is known, the energy can be computed from the formula

$$
\begin{aligned}
E & =\mathcal{M} \sum_{j=1}^{N_{H}} \cosh h_{j}-2 \mathcal{M} \sum_{j=1}^{N_{S}} \cosh y_{j} \\
& -\mathcal{M} \sum_{j=1}^{M_{C}} \cosh c_{j}+\mathcal{M} \sum_{j=1}^{M_{W}}\left(\cosh w_{j}\right)_{I I} \\
& -\mathcal{M} \int_{-\infty}^{\infty} \frac{d x}{2 \pi} 2 \Im m\left[\sinh (x+i \eta) \log \left(1+(-1)^{\delta} e^{i Z(x+i \eta)}\right)\right],
\end{aligned}
$$

with a similar expression for the momentum.

We start our investigation by recalling some facts about the UV analysis of the spectrum of the NLIE, i.e. the consideration of the so called kink equation and its consequences. The UV limit of sG theory is $c=1$ conformal field theory (CFT) with a compactification radius $R$ which is related to the six-vertex anisotropy $\gamma$ by

$$
R^{-1}=\sqrt{2\left(1-\frac{\gamma}{\pi}\right)}
$$

and it is often convenient to introduce the parameterf

$$
p=\frac{\pi}{\gamma}-1
$$

The primary fields are vertex operators $V_{(n, m)}$ with conformal weights

$$
\Delta_{(n, m)}^{ \pm}=\frac{1}{2}\left(\frac{n}{R} \pm \frac{1}{2} m R\right)^{2}
$$

\footnotetext{
${ }^{4} p>1$ (i.e. $0<\gamma<\pi / 2$ ) corresponds to the repulsive while $p<1$ (i.e. $\pi / 2<\gamma<\pi$ ) to the attractive regime of $\mathrm{sG}$ theory. The $n$th breather threshold is at $p=1 / n$.
} 
where $m \in \mathbb{Z}$ is the winding number (which is conserved in the off-critical theory and is identified with the sG topological charge $Q$ ) and $n$ is the field momentum. The relation (4) means that we identify the perturbing operator with $V_{(1,0)}+V_{(-1,0)}$.

We recall some of the results of ref. [13]. In the UV limit $l \rightarrow 0$ the sources can be classified into three types: their position can remain finite ("central"), or they can move towards the two infinities as $\pm \log \frac{2}{l}$ ("left/right movers"). We denote the number of right/left moving holes by $N_{H}^{ \pm}$and similarly we introduce the numbers $N_{S}^{ \pm}, M_{C}^{ \pm}$and $M_{W}^{ \pm}$.

We introduce the partial spins $S^{+}, S^{-}$by the definition:

$$
S^{ \pm}=\frac{1}{2}\left[N_{H}^{ \pm}-2 N_{S}^{ \pm}-M_{C}^{ \pm}-2 M_{W}^{ \pm} \theta(\pi-2 \gamma)\right]
$$

The expression for the conformal weights is

$$
\Delta_{ \pm}= \pm\left(I_{H}^{ \pm}-2 I_{S}^{ \pm}-I_{C}^{ \pm}-I_{W}^{ \pm}\right)+\frac{\Sigma_{ \pm}}{2 \pi}+\frac{\omega_{ \pm}^{2}}{16 \pi^{2}(1-\gamma / \pi)}
$$

where

$$
\omega_{ \pm}= \pm 2(\pi-2 \gamma)\left(S-2 S^{ \pm}\right)-2(\pi-\gamma)\left(\delta+2 k_{ \pm}\right)
$$

$k_{ \pm}$are integers determined by the condition $-\pi \leq \omega_{ \pm} \leq \pi$,

$$
\Sigma_{ \pm}=-4 S^{ \pm}\left(S-S^{ \pm}\right) \frac{\pi-2 \gamma}{1-\gamma / \pi}+2 \pi q_{W}^{ \pm}
$$

and we introduced the following notation for the sums of quantum numbers of left/right moving sources of the different types

$$
I_{H}^{ \pm}=\sum_{j=1}^{N_{H}^{ \pm}} I_{h_{j}}^{ \pm}, I_{C}^{ \pm}=\sum_{j=1}^{M_{C}^{ \pm}} I_{c_{j}}^{ \pm}, I_{W}^{ \pm}=\sum_{j=1}^{M_{W}^{ \pm}} I_{w_{j}}^{ \pm} \text {and } I_{S}^{ \pm}=\sum_{j=1}^{N_{S}^{ \pm}} I_{y_{j}}^{ \pm}
$$

$\left(q_{W}^{ \pm}\right.$is an integer or half-integer depending on the configuration of the wide roots, which is best calculated case by case).

From (5) we obtain the following expressions:

$$
\begin{gathered}
m=2 S \\
2 n=\left(\delta+2 S+M_{s c}\right) \bmod 2
\end{gathered}
$$

where $M_{s c}$ is the number of selfconjugate wide roots. Since $S \in \mathbb{Z}$ we have access only to sectors with even value of the topological charge $Q=m$. In [13], the selection rule for sG states was shown to be

$$
\left(\delta+M_{s c}\right) \bmod 2=0 .
$$

Now let us consider putting $S$ half-integer in (8) and remember that in order to have the sG with periodic boundary conditions we need $n \in \mathbb{Z}$. Thus we obtain the rule

$$
\left(\delta+M_{s c}\right) \bmod 2=1 .
$$

From (2) we see that if $S \in \mathbb{Z}+\frac{1}{2}$ then $N_{H}$ must be odd (given that the number of close complex roots $M_{C}$ is always even). Therefore to describe such states we put an odd number of 
hole sources in the NLIE. This extension of the equation cannot be derived from the light-cone lattice approach, since the latter only allows for even number of holes. In the following we will take it as a conjecture and give arguments in support.

Let us now present examples of UV dimensions computed from the NLIE for some simple states. For the state with one hole with quantum number $I=0$, using $\delta=1$ we obtain the conformal dimensions

$$
\Delta^{ \pm}=\frac{1}{8 R^{2}}
$$

corresponding to the vertex operator $V_{(0,1)}$. This operator can be identified as the UV limit of the one-soliton field as it is well known 15 .

Consider now the case of three holes, with quantum numbers $I_{1}=1, I_{2}=0$ and $I_{3}=-1$. The conformal dimension we obtain corresponds to the vertex operator $V_{(0,3)}$ as expected. If we choose other values of quantum numbers different from this "minimal" choice, we obtain descendents of vertex operators $V_{(n, 3)}$, where $n \in \mathbb{Z}$ depends on the number of holes moving to the left and to the right.

As a last example, let us take three holes with quantum numbers $I_{1}=1, I_{2}=0$ and $I_{3}=-1$, but we add a close pair with quantum number 0 for both complex roots. We obtain the conformal weights

$$
\Delta^{ \pm}=\frac{1}{8 R^{2}}+1
$$

which is the first nonchiral descendent of $V_{(0,1)}$.

Using the above results, we formulate our conjecture as follows: the sG states with odd value of topological charge correspond to taking an odd number of holes and choosing the quantisation rule $\delta$ in accordance with (9). In the next section we shall see that using this rule we get scaling functions which are in excellent agreement with numerical results obtained from the $c=1$ Truncated Conformal Space (TCS) method.

\section{Numerical comparison between the NLIE and TCS}

In this section we present the numerical results which give further support to the conjecture on the identification of $\mathrm{sG}$ states with odd topological charge. In particular, we compare the NLIE prediction to numerical results obtained from the TCS method for the lowest energy levels.

We start with the one-soliton state, which is conjectured to be a one-hole configuration with integer quantisation. Figure 1 shows the comparison graphically, while table 1 gives an idea about the numerical magnitude of the difference. Note that the deviations are extremely small for small values of $l$ and they grow with the volume, exactly as expected for truncation errors.

Next we take the two lowest energy levels corresponding to states of three solitons with the same charge (i.e. three holes with quantum numbers $0, \pm 1$ and $0, \pm 2$, respectively). Here we only present the energy plot (figure 2). The numerical deviations are exactly of the same magnitude as in the case of the one-soliton state.

The value of the coupling constant at which the above data are presented lies in the attractive regime where the TCS method is free of UV divergences 112, 13 and therefore we can compare absolute energy levels, after subtracting the linear bulk energy term

$$
\frac{E_{b u l k}}{M}=B l, B=-\frac{1}{4} \tan \frac{\pi p}{2},
$$




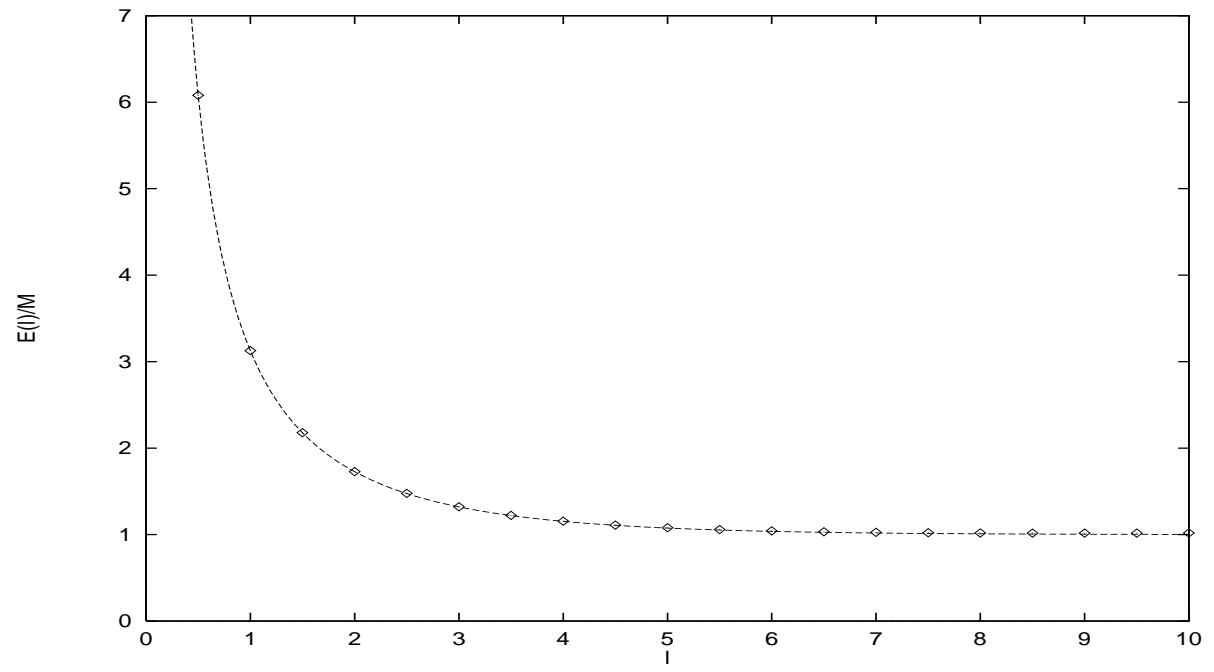

Figure 1: Comparison of the results for the one-soliton state coming NLIE and TCS at $p=\frac{2}{7}$. The continuous line is the NLIE result, while the diamonds represent the TCS data.

\begin{tabular}{|c|c|c|c|}
\hline 1 & NLIE & TCS & Relative deviation \\
\hline \hline .5 & 6.080571 & 6.08062 & 0.000008 \\
\hline 1 & 3.126706 & 3.12685 & 0.00005 \\
\hline 1.5 & 2.177411 & 2.17791 & 0.0002 \\
\hline 2 & 1.727224 & 1.72776 & 0.0003 \\
\hline 2.5 & 1.475004 & 1.47593 & 0.0006 \\
\hline 3 & 1.320353 & 1.32168 & 0.001 \\
\hline 4 & 1.153188 & 1.15548 & 0.002 \\
\hline 5 & 1.075376 & 1.07908 & 0.003 \\
\hline
\end{tabular}

Table 1: Numerical comparison of the energy levels predicted by the NLIE to the TCS data for the case depicted in figure 1. The NLIE data are exact to the precision shown. 


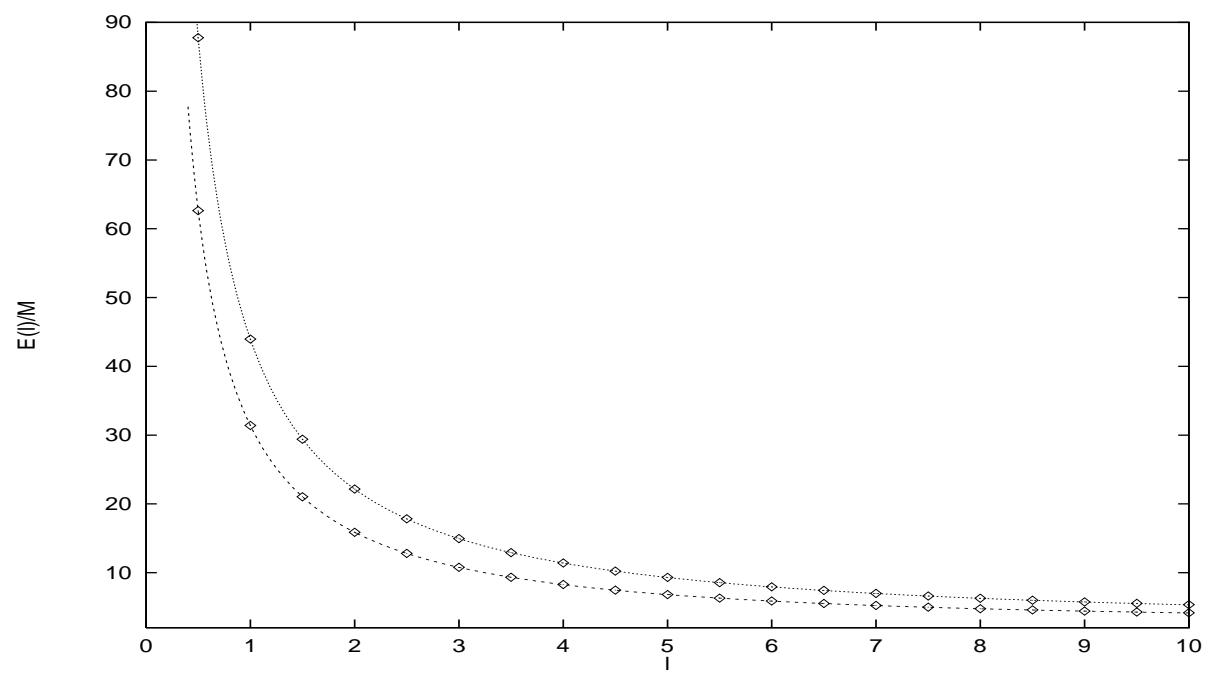

Figure 2: The energies as a function of the volume for first two three-soliton states coming from NLIE and TCS at $p=\frac{2}{7}$. The continuous lines are the NLIE result, while the diamonds represent the TCS data.

\begin{tabular}{|c|c|c|c|}
\hline 1 & NLIE & TCS & Relative deviation \\
\hline \hline .5 & 25.27656 & 25.3398 & 0.0025 \\
\hline 1 & 12.79207 & 12.8854 & 0.007 \\
\hline 1.5 & 8.674948 & 8.79114 & 0.01 \\
\hline 2 & 6.647771 & 6.78091 & 0.02 \\
\hline 2.5 & 5.455906 & 5.60033 & 0.026 \\
\hline 3 & 4.680766 & 4.83188 & 0.03 \\
\hline 4 & 3.751729 & 3.90497 & 0.04 \\
\hline 5 & 3.231486 & 3.37598 & 0.045 \\
\hline
\end{tabular}

Table 2: Numerical comparison of the energy levels predicted by the NLIE to the TCS data for the case depicted in figure 3 .

which is predicted by TBA [16] and also by the NLIE [6], from the TCS data.

The state with three holes and one complex pair can be most easily investigated in the repulsive regime, since it changes its nature in the attractive regime where the complex roots behave in a more complicated way. The result is depicted in figure 3. As in the repulsive regime the TCS is UV divergent, one can only compare relative energy levels, and as we are in the sector with unit topological charge, the natural way is to normalize the energy levels to the one-soliton state, which is the ground state of the $Q=1$ sector. Although this cannot be directly seen from figure 3, it is clear from the numbers presented in table 2 that the truncation errors are much larger even after subtracting the UV divergences. The same phenomenon was observed in our earlier works [12, 13].

\section{Scaling functions in the massive Thirring model}

Here we formulate another conjecture concerning the finite size energy levels of the massive Thirring model. Of the two quantum numbers $n, m$ specifying a vertex operator $V_{(n, m)}, m$ can 


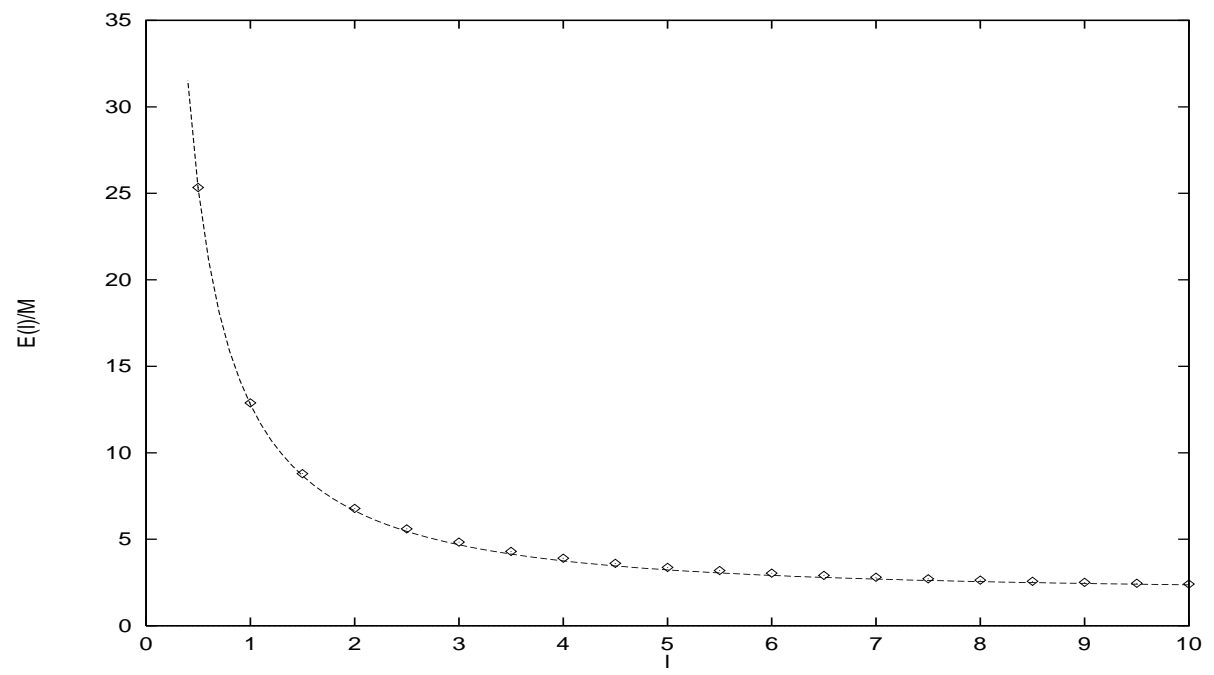

Figure 3: The NLIE prediction for the state with three holes (with quantum numbers $-1,0$ and 0 ) and a complex pair, at $p=1.5$, compared to TCS data. Both are normalized to the ground state of the $Q=1$ sector, i.e. the one-soliton state, to get free of the UV divergent part of the TCS data.

be identified with the sG topological charge and takes integer values, while $n$ is not conserved in the massive theory. Generally, one can introduce twisted boundary conditions, for which

$$
n \in \mathbb{Z}+\frac{\nu}{2 \pi},
$$

where the parameter $\nu$ lies between 0 and $2 \pi$. Such boundary conditions can be realised by adding a constant term $\alpha$ to the right hand side of the NLIE which is related to $\nu$ as

$$
\nu=\alpha-\pi \delta \bmod 2 \pi .
$$

Here we will need only the cases $\nu=0$ or $\pi$, which are realised by taking $n \in \mathbb{Z} / 2$. For convenience, we divide the Hilbert space into four sectors, given by

$$
\begin{aligned}
& \mathcal{H}_{I}=\bigoplus\left\{\mathcal{F}_{(n, m)}: n \in \mathbb{Z}, m \in 2 \mathbb{Z}\right\}, \\
& \mathcal{H}_{I I}=\bigoplus\left\{\mathcal{F}_{(n, m)}: n \in \mathbb{Z}, m \in 2 \mathbb{Z}+1\right\}, \\
& \mathcal{H}_{I I I}=\bigoplus\left\{\mathcal{F}_{(n, m)}: n \in \mathbb{Z}+\frac{1}{2}, m \in 2 \mathbb{Z}+1\right\}, \\
& \mathcal{H}_{I V}=\bigoplus\left\{\mathcal{F}_{(n, m)}: n \in \mathbb{Z}+\frac{1}{2}, m \in 2 \mathbb{Z}\right\},
\end{aligned}
$$

where $\mathcal{F}_{(n, m)}$ denotes the Fock module corresponding to the vertex operator $V_{(n, m)}$. According to [15], the sG Hilbert space (for periodic boundary conditions) can be identified with $\mathcal{H}_{I} \oplus \mathcal{H}_{I I}$ while that of $\mathrm{mTh}$ is $\mathcal{H}_{I} \oplus \mathcal{H}_{I I I}$. Note that these two spaces are related to each other by twisting the boundary conditions. The corresponding two algebras are two different maximal closed local operator algebras, which are essentially unique (see comments at the end of the section) at generic values of the compacitification radius $R . \mathcal{H}_{I V}$ never appears as a local sector in $\mathrm{sG} / \mathrm{mTh}$ theory. Using the results from the UV limit of the NLIE (8), we see that the massive Thirring model is selected by (putting $\alpha=0$ )

$$
\delta=M_{s c} \bmod 2 .
$$




\begin{tabular}{|c|c|c|c|}
\hline 1 & NLIE & TCS & Relative deviation \\
\hline \hline 3.0 & 1.549935 & 1.55081 & 0.0006 \\
\hline 3.5 & 1.415153 & 1.41657 & 0.001 \\
\hline 4.0 & 1.321588 & 1.32357 & 0.0015 \\
\hline 4.5 & 1.254597 & 1.25722 & 0.002 \\
\hline 5.0 & 1.205454 & 1.20881 & 0.003 \\
\hline
\end{tabular}

Table 3: The comparison between the NLIE results for the energy of the one-fermion state and the TCS data for the range $3.0<l<5.0$. The coupling constant is $p=\frac{2}{7}$ and the hole quantum number was chosen $I=\frac{1}{2}$.

As an example, let us consider the one-hole state with half-integer quantisation and quantum number $I=\frac{1}{2}$. Using the general formulae we compute the UV dimensions

$$
\Delta^{ \pm}=\frac{1}{2}\left(\frac{1}{2 R} \pm \frac{R}{2}\right)^{2}
$$

which corresponds to the vertex operator $V_{(1 / 2,1)}$. If we choose instead $I=-\frac{1}{2}$, we obtain the UV limit $V_{(-1 / 2,1)}$. These are exactly the positive charge components of the (Dirac) fermion field in the $c=1 \mathrm{CFT}$ and so we can identify the one-hole configuration with the one-fermion state of the massive Thirring model. One can also compute the dimensions of more general configurations and verify that they describe states in $\mathcal{H}_{I} \oplus \mathcal{H}_{I I I}$.

The iterative solution of the NLIE presents serious difficulties when the arrangement of the source terms is not symmetric under $\vartheta \rightarrow-\vartheta$. The number of steps generally increases when $l$ decreases. This can be prevented by making a re-antisymmetrization of the counting function computed at each step, which however can be done only for symmetric source configurations. For $\mathrm{mTh}$ states of odd charge, the source configurations are never symmetric. In the case of the one-fermion state, the iteration slows down too much to reach values of $l$ less than 3.0. The solution of this problem may necessitate the introduction of some fundamentally new numerical algorithm to solve the NLIE and prevents us from presenting a numerical comparison to TCS data of such mTh states for the time being. The results available for regions of $l$ where the iteration converges fast enough (see table 3) show an agreement with TCS similar to the one presented for sG states in the previous section (cf. table 1).

Our selection rule (10) takes into account that, as described in our previous paper, we redefined the source term for the self-conjugated roots (see section 3.2 of [13]). If one keeps the form of the source terms as they are derived from the lattice, one can summarize the selection rules for the even and the odd sector of mTh model in the following simple form:

$$
\delta=0 \bmod 2 .
$$

With the same convention, the selection rule for the sine-Gordon model becomes

$$
\delta=2 S \bmod 2 .
$$

Let us make a remark on the uniqueness of the maximal local operator algebra. The two operator algebras correspoding to the bosonic theory sG and the fermionic theory $\mathrm{mTh}$

$$
\begin{aligned}
& \mathcal{A}_{b}=\left\{V_{(n, m)}: n \in \mathbb{Z}, m \in \mathbb{Z}\right\} \\
& \mathcal{A}_{f}=\left\{V_{(n, m)}: n \in \mathbb{Z}, m \in \mathbb{Z}+n / 2\right\}
\end{aligned}
$$


are the only ones at a generic value of the compactification radius $R$ in the sense that any other maximal local operator algebras can be mapped into them by redefining $R$ by multiplication with an integer 15. However, the identification of the perturbation operator is not unique: it can be taken to be any of $V_{k, 0}+V_{-k, 0}$ with $k$ integer. This would change the value of $R$ in (4) by a factor of $k$. If we map these theories back to perturbations by $V_{1,0}+V_{-1,0}$ by redefining $R$ to the value in (雨), we obtain the following other local operator algebras:

$$
\begin{aligned}
& \mathcal{A}_{b}^{(k)}=\left\{V_{(n / k, k m)}: m \in \mathbb{Z}, n \in \mathbb{Z}\right\} \\
& \mathcal{A}_{f}^{(k)}=\left\{V_{(n / k, k m)}: m \in \mathbb{Z}, n \in \mathbb{Z}+m / 2\right\}
\end{aligned}
$$

This is the complete list of possible local theories as $k$ runs over all positive integers. Note that $\mathrm{sG} / \mathrm{mTh}$ corresponds to choosing $k=1$. Another interesting theory is the one defined by $\mathcal{A}_{b}^{(2)}$ with the corresponding Hilbert space given by $\mathcal{H}_{I} \oplus \mathcal{H}_{I V}$. At the Kosterlitz-Thouless point $\gamma=0$ where the perturbation becomes marginal this describes the current-current perturbation of a level-1 $S U(2)$ WZNW model, which is nothing else than the SU(2) GrossNeveu theory. Note that the states with odd topological charge are absent from the local sector. This is due to the fact that the elementary excitation is a "kink" with fractional Lorentz spin $1 / 4$. To describe the kink state, created by the operator $V_{ \pm 1 / 4, \pm 1}$ in the UV, one has to twist the odd sector NLIE by $\alpha= \pm \pi / 2$. This sort of nonlocality is a general property of the models based on $\mathcal{A}_{b, f}^{(k)}$ which is why they were called "kink" theories in 115 .

\section{Conclusions}

In this letter we have proposed a generalization of the NLIE to the odd charge sectors of sG and $\mathrm{mTh}$ models. The conjecture has been tested against the data coming from a TCS diagonalization of the $c=1$ perturbed CFT Hamiltonian that is expected to give a description of $\mathrm{sG} / \mathrm{mTh}$ models. The agreement is excellent and gives strong evidence for our conjecture, which is also supported by the fact that the proposal is the most obvious and natural generalization of the even sector NLIE. Further evidence is given by the UV analysis reproducing correctly the conformal dimensions of the states to be found in such sectors, and in particular by the successful identification of the single sG soliton state as well as the two components of the mTh fermion.

It is interesting to notice that the NLIE framework together with this proposal allows for a complete treatment of both $\mathrm{sG}$ and $\mathrm{mTh}$ Hilbert spaces. The two models, according to the interpretation of ref. [15], correspond to two subspaces of the same space, identified in the UV limit by the action of two different maximal local subalgebra of conformal operators. One leads to a counting of states described by the modular invariant partition function, while the other corresponds to the $\Gamma_{2}$-invariant one. mTh model corresponds to sG with twisted boundary conditions and vice-versa. All these features can now be controlled also away from UV criticality, thanks to the NLIE approach that we have in a sense "completed" with the present extension to odd charge sectors.

Of course one could think to investigate different local UV operator algebras, identifying other models, e.g. the algebras $\mathcal{A}_{b, f}^{(k)}$. These are controllable on the level of the NLIE by choosing appropriately the twist parameter $\alpha$ implementing boundary conditions. Thus one could explore, e.g., the formulation of the deformed $S U(2)$ Gross-Neveu model which is the QFT having $\mathcal{A}_{b}^{(2)}$ as local operator algebra away from the Kosterlitz-Thouless point. Other 
interesting QFTs arise at special ("rational") values of the radii, like the $\Phi_{(1,3)}$ perturbations of minimal models. Notice that in the language of ref. [16], all these models are just "projections" onto different local sectors, of the same Hilbert space of sG model on the cylinder, if $\alpha$-sectors are meant to be included in the Hilbert space from the beginning.

It would be interesting, as a further development, to propose a modified light-cone lattice formulation of $\mathrm{sG} / \mathrm{mTh}$, leading to Bethe equations that allow the treatment of odd number of holes. A proposal in this respect has been put forward by Destri and Segalini [17], in order to avoid the nonlocality present in the original light-cone lattice approach [18\|. This nonlocality is manifested both in the lattice Hamiltonian and in the construction of the Thirring fermion field on the lattice. The fermionic light-cone lattice model has Bethe equations that allow for odd number of holes and look very similar to the ones derived from the "traditional" light-cone approach. However, since in this framework the fermion field is represented locally on the lattice, there appears the well-known doubling of fermionic species. The doublers are shown to decouple from the physical fields in the continuum limit of the Hamiltonian. In order to give a derivation of the NLIE starting from the fermionic formalism it is necessary to follow this decoupling in the Bethe equations themselves, which is out of the scope of this paper. We would just like to point out that the rule of half-integer Bethe quantisation in the mTh model is in complete accord with their fermionic lattice model.

Acknowledgements - We are indebted to C. Destri for useful discussions. This work was supported in part by NATO Grant CRG 950751, by European Union TMR Network FMRXCT96-0012 and by INFN Iniziativa Specifica TO12. G. T. has been partially supported by the FKFP 0125/1997 and OTKA T016251 Hungarian funds.

\section{References}

[1] M. Lüscher, Comm. Math. Phys. 104 (1986) 177-206.

M. Lüscher, Comm. Math. Phys. 105 (1986) 153-188.

[2] R. Guida and N. Magnoli, Phys. Lett. B411 (1997) 127-133, hep-th/9706017.

[3] V.P. Yurov and A.B. Zamolodchikov, Int.J.Mod.Phys. A5 (1990) 3221-3246.

[4] Al.B. Zamolodchikov, Nucl. Phys. B342 (1990) 695-720.

[5] C. Destri and H.J. De Vega, Phys. Rev. Lett. 69 (1992) 2313-2317.

[6] C. Destri and H.J. De Vega, Nucl. Phys. B438 (1995) 413-454, hep-th/9407117.

[7] A. Klümper and P.A. Pearce, J. Stat. Phys. 64 (1991) 13;

A. Klümper, M. Batchelor and P.A. Pearce, J. Phys. A24 (1991) 3111.

[8] P. Dorey and R. Tateo, Nucl. Phys. B482 (1996) 639-659, hep-th/9607167.

P. Dorey and R. Tateo, Nucl. Phys. B515 (1998) 575-623, hep-th/9706140.

[9] V.V. Bazhanov, S.L. Lukyanov and A.B. Zamolodchikov, Nucl. Phys. B489 (1997) 487531, hep-th/9607099.

[10] D. Fioravanti, A. Mariottini, E. Quattrini and F. Ravanini, Phys. Lett. B390 (1997) 243-251, hep-th/9608091. 
[11] C. Destri and H. De Vega, Nucl. Phys. B504 (1997) 621-664, hep-th/9701107.

[12] G. Feverati, F. Ravanini and G. Takács: Truncated Conformal Space at $c=1$, Nonlinear Integral Equation and Quantisation Rules for Multi-Soliton States, preprint DFUB-98-04, hep-th/9803104, Physics Letters B in press.

[13] G. Feverati, F. Ravanini and G. Takács: Nonlinear Integral Equation and Finite Volume Spectrum of Sine-Gordon Theory, preprint DFUB-98-10, hep-th/9805117.

[14] Al. B. Zamolodchikov, Nucl. Phys. B432 (1994) 427-456, hep-th/9409108.

[15] T. Klassen and E. Melzer, Int. J. Mod. Phys. A8 (1993) 4131-4174, hep-th/9206114.

[16] Al.B. Zamolodchikov, Int. J. Mod. Phys. A10 (1995) 1125-1150.

[17] C. Destri and T. Segalini, Nucl. Phys. B455 (1995) 759, hep-th/9506120.

[18] C. Destri and H.J. De Vega, Nucl. Phys. B290 (1987) 363-391. 